\title{
Could some meteoritic stardust have originated from the winds of post-AGB stars and planetary nebula nuclei?
}

\author{
Joelene Buntain $^{1}$, Maria Lugaro ${ }^{1}$ and Amanda Karakas ${ }^{2}$ \\ ${ }^{1}$ Monash Center for Astrophysics, Monash University, \\ Wellington Road, Clayton 3800, Australia \\ email: Joelene.Buntain@monash.edu Maria.Lugaro@monash.edu \\ ${ }^{2}$ RSAA, Australian National University, \\ Mount Stromlo Observatory, Canberra, Australia \\ email: akarakas@mso.anu.edu.au
}

\begin{abstract}
After very dense and slow winds erode the outer layer of an asymptotic giant branch (AGB) star down to a thin H-rich layer $\left(\simeq 10^{-3} \mathrm{M}_{\odot}\right)$ the star becomes a post-AGB star and evolves at constant luminosity towards hotter temperatures. It may then becomes a planetary nebula nucleus (PNN) at the centre of a planetary nebula (PN). During these phases, the thin $\mathrm{H}-$ rich surface layer of the star is eroded by winds. Stardust oxide and silicate grains are recovered from meteorites. The origin of the "Group II grains" that show enrichments in ${ }^{17} \mathrm{O}$ and depletions in ${ }^{18} \mathrm{O}$ is currently explained by invoking the occurrence of some kind of extra-mixing process in AGB stars. We suggest instead that these grains originated from the winds of post-AGB stars and PNN. These winds show the signature of H-burning. We will do this by comparing our predictions from stellar models to the compositions observed in Group II stardust oxide and silicate grains. We find that the composition of the thin H-rich layer lost in the post-AGB and PNN winds is close to that of Group II grains, however the match with the Al ratios needs to be improved. Considering the uncertainities in the ${ }^{25} \mathrm{Mg}$ and ${ }^{26} \mathrm{Al}$ proton capture rates may be helped in this respect.
\end{abstract}

Keywords. circumstellar matter, stars: AGB and post-AGB, stars: winds, outflows

\section{Introduction}

Stardust grains are tiny specks of dust recovered from primitive meteorites and analysed in the laboratory as solid samples of stars. Stardust oxide and silicate grains are classified into four groups (Nittler et al. 1997): Group I grains show the signature of the first dredge-up and are believed to have originated from red giant and AGB stars. Group II grains show deficit in ${ }^{18} \mathrm{O}$, enrichment in ${ }^{17} \mathrm{O}$ and excess in ${ }^{26} \mathrm{Al}$. The current explanation for their composition involves the presence of some kind of extra-mixing process in AGB stars. Group III grains origin is unclear. Group IV grains show the signature of an origin from supernovae. We propose an alternative explanation for the composition of Group II grains as originating from the winds of post-AGB stars and PNN. The observational signature of the dust forming around PNN has been inferred by Cohen \& Barlow (1974) on the basis of infrared observations.

\section{Method and results}

To look at the composition of post-AGB and PNN winds we took 3 stellar models (1, 1.25 and $1.5 \mathrm{M}_{\odot}, \mathrm{Z}=0.014$ )and selected the deepest $10^{-3} \mathrm{M}_{\odot}$ H-rich region of each star at 

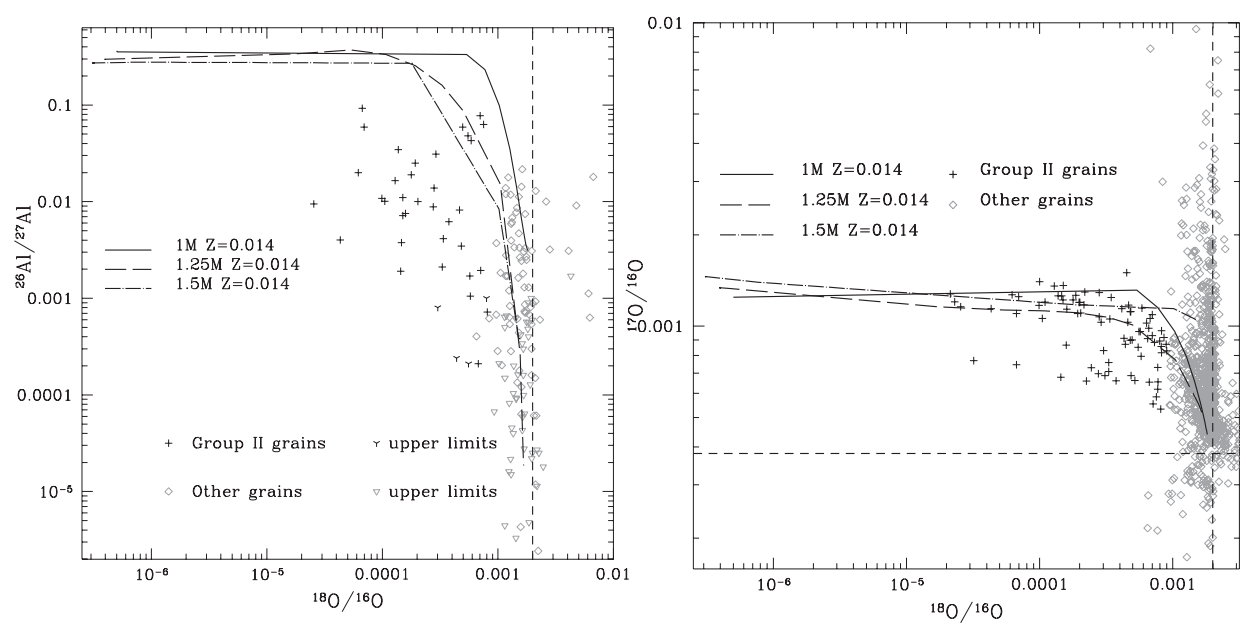

Figure 1. The ${ }^{26} \mathrm{Al} /{ }^{27} \mathrm{Al}$ versus ${ }^{17} \mathrm{O} /{ }^{16} \mathrm{O}$ isotopic ratios from the models compared to the stardust data. Each line is a different model and represents the computed isotopic ratios corresponding to a mass of

Figure 2. The ${ }^{17} \mathrm{O} /{ }^{16} \mathrm{O}$ versus ${ }^{18} \mathrm{O} /{ }^{16} \mathrm{O}$ isotopic ratios from the models compared to the stardust grain data. Lines are the same as in Figure 1. $10^{-3} \mathrm{M} \odot$ in the deepest H-rich layer of the star. The dashed lines are the solar values.

the end of the computed AGB evolution as this would represent the material lost in the post-AGB and PNN winds. The isotopic compositions of this region show the signature of H-burning, which is thus imprinted in the material eroded by the fast stellar winds of post-AGB and PNN. In Figures 1 and 2 we compare this composition to that observed in Group II grains. We find that the oxygen and aluminium isotopic ratios are close to those of the Group II grains. The match with the aluminium ratios may be improved considering the large uncertainities in the ${ }^{25} \mathrm{Mg}$ and ${ }^{26} \mathrm{Al}$ proton capture rates.

\section{Discussion}

A problem with this scenario is that the mass involved $(0.1 \%$ of the total mass lost during the life of the star) is too small to reproduce the frequency of the Group II grains, because the ratio of Group II grains to Group I grains is $\simeq 10 \%$. Furthermore, the observed gas-to-dust ratios of PNN is $10^{-4}$ times smaller than that of AGB stars. However, the stardust grains considered here have a size of $\simeq 1 \mu \mathrm{m}$, while those observed to form in O-rich AGB winds are at most $\simeq 0.3 \mu \mathrm{m}$ (Jura 1996). Formation in a disk would allow more time for the grains to grow. Disk structures associated with large grains have been observed around binary post-AGB stars and around PNN (Murakawa et al. 2010 and references therein). Thus, we suggest that material from winds of post-AGB stars and PNN may have greatly contributed to the observed number of stardust grains.

\section{References}

Cohen, M. \& Barlow M. J. 1974, ApJ, 193, 401

Jura, M. 1996, ApJ, 472, 806

Murakawa, K., Ueta, T., \& Meixner, M. 2010, A\& A, 510, A30

Nittler, L. R., Alexander, C., Gao, X, Walker, R., \& Zinner, E. 1997, NuPhA, 621, 113 